\title{
Analysis of astrovirus transmission pathways in a free-ranging fission-fusion colony of Natterer's bats (Myotis nattereri)
}

\author{
Veronika M. Zeus ${ }^{1}$ (D) $\cdot$ Arian Köhler ${ }^{2} \cdot$ Christine Reusch $^{1} \cdot$ Kerstin Fischer $^{2} \cdot$ Anne Balkema-Buschmann $^{2}$. \\ Gerald Kerth ${ }^{1}$
}

Received: 7 March 2020 / Accepted: 6 November 2020 / Published online: 17 November 2020

(C) The Author(s) 2020

\begin{abstract}
Bats are a diverse and widespread order of mammals. They fulfill critical ecosystem roles but may also act as reservoirs and spreaders for zoonotic agents. Consequently, many recent studies have focused on the potential of bats to spread diseases to other animals and to humans. However, virus transmission networks within bat colonies remain largely unexplored. We studied the detection rate and transmission pathway of astroviruses in a free-ranging Natterer's bat colony (Myotis nattereri) that exhibits a high fission-fusion dynamic. Based on automatic roost monitoring data of radio-frequency identification tagged bats, we assessed the impact of the strength of an individual's roosting associations with all other colony members (weighted degree), and the number of roost sites (bat boxes) an individual used — both being proxies for individual exposure risk —on the detected presence of astrovirus-related nucleic acid in individual swab samples. Moreover, we tested to which degree astrovirus sequence types were shared between individuals that frequently roosted together, as proxy for direct transmission risk, and between bats sharing the same roost sites in close temporal succession, as proxy for indirect transmission risk. Neither roosting associations nor the number of different roost sites had an effect on detected virus presence in individual bats. Transmission network data suggest that astroviruses are transmitted both via direct and indirect contact, implying that roost sites pose a risk of astrovirus infection for several days after the bats leave them. Our study offers novel insights in the presence and transmission of viruses within social networks of bat colonies.
\end{abstract}

\section{Significance statement}

Bats provide many ecosystem services but have moved into the focus of virological research as potential carriers of zoonotic disease agents. However, the sparse information available about virus transmission within bat colonies is solely based on simulated transmission data. In this field study, we examined the daily roosting behavior in a wild bat colony in relation to the presence of viruses in individual colony members. Our findings suggest that astroviruses are transmitted by direct contact and via contaminated roost sites. Bats typically defecate in their roost sites, and astroviruses can remain infectious in feces for several days. The here observed virus diversity and roosting behavior suggest that bats can contract astroviruses even if they use contaminated roost sites days after infected individuals have left. This study provides first-time insights in the transmission of astroviruses within bat colonies in the wild.

Keywords Bats $\cdot$ Social network $\cdot$ Astrovirus $\cdot$ Transmission $\cdot$ Roost site use

Communicated by G. S Wilkinson

Veronika M. Zeus

veronika.zeus@gmail.com

1 Zoological Institute and Museum, Greifswald University, Loitzer Str. 26, 17489 Greifswald, Germany

2 Institute of Novel and Emerging Infectious Diseases, Friedrich-Loeffler-Institut, Südufer 10, 17493 Greifswald-Insel Riems, Germany

\section{Introduction}

Living in social groups provides multiple benefits to the individual group members (Krause and Ruxton 2002) but at the same time increases the risk of exposure to diseases, as large groups will typically harbor more pathogens than small groups or solitary individuals (Loehle 1995). Pathogens such as viruses can be transmitted within the group via physical contact (direct transmission) and/or contaminated shared 
resources (indirect transmission) (Taylor et al. 2001). Thus, individual exposure risk may depend on both the association behavior of group members and on joint resource use, even if individuals do not meet directly because they use the resource at different times.

Bats are among the most gregarious mammals and often form colonies where many individuals roost in close contact (Kerth 2008). In many bat species, the colonies frequently split into temporary subgroups that use a number of separate roost sites (Kerth and König 1999; O’Donnell 2000; Garroway and Broders 2007; Popa-Lisseanu et al. 2008). A behavior called "fissionfusion" that also occurs in many other mammalian orders such as social carnivores, cetaceans, ungulates, primates, and proboscideans (e.g., Archie et al. 2006; Sundaresan et al. 2007; Amici et al. 2008; Smith et al. 2008; Parra et al. 2011; Carter et al. 2013). Furthermore, bats provide a multitude of different ecosystem services with both ecological and economic value (Kunz et al. 2011). They play an important role in arthropod control, pollination, and seed dispersal but are also culturally anchored as provider of consumptive goods (e.g., guano, food source, traditional medicine; Kunz et al. 2011). Not least due to their use by humans, they have come to the fore as vectors and reservoir hosts for a number of viruses, including those with zoonotic potential (Calisher et al. 2006; Omatsu et al. 2007; Chan et al. 2013; Luis et al. 2013; Klimpel and Mehlhorn 2014). However, while many recent studies have focused on their possible role of spreading diseases to other animals and to humans (Wood et al. 2012; Plowright et al. 2015), virus transmission networks within bat colonies remain largely unexplored, and the few available studies rely on simulated transmission data (Kashima et al. 2013; Webber et al. 2016).

Astroviruses are small, nonenveloped viruses with an icosahedral capsid and a diameter of 28 to $35 \mathrm{~nm}$. They have a singlestranded, positive sense ribonucleic acid (RNA) genome with no $5^{\prime}$ cap, three open reading frames (ORF1a, ORF1b, and ORF2), and a poly(A) tail at the $3^{\prime}$ end. While the overlapping ORF1a and $1 \mathrm{~b}$ encode the viral protease and RNA-dependent RNA polymerase (RdRp) respectively, ORF2 encodes the capsid proteins (Cortez et al. 2017). Astrovirus infections are common in numerous avian and mammalian species including humans (Moser and Schultz-Cherry 2005; De Benedictis et al. 2011) and bats (Fischer et al. 2016, 2017; Halczok et al. 2017). After the discovery of human astroviruses, an exclusive tropism to the digestive system and fecal shedding has been assumed for many years, as astroviruses typically cause diarrhea in humans and domestic species (Moser and Schultz-Cherry 2005). In the meantime, it has become clear that astroviruses can also affect many other organs, including the respiratory (Padmanabhan and Hause 2016) and urinary systems (Imada et al. 2000; Zhao et al. 2011; Singh et al. 2016) as well as the central nervous system (Blomström et al. 2010; Li et al. 2013; Bouzalas et al. 2014; Schlottau et al. 2016; Pfaff et al. 2017). It can therefore be assumed that the virus is shed by more than one route.
However, like most other viral infections in bats, individuals carrying astrovirus-related RNA appear healthy (Calisher et al. 2006; Chu et al. 2008; Baker et al. 2013; Kemenesi et al. 2014; Fischer et al. 2017; Amoroso et al. 2018). Despite the high prevalence of astroviruses among various bat species, to our knowledge, nothing is known about the persistence of astroviruses in the bat host or the environment, although human astroviruses can persist for several days on inanimate surfaces (Abad et al. 2001). Neither have astrovirus infection routes in bats been in the focus of research, as mentioned above. However, due to the observed stability of astroviruses in the environment (Abad et al. 2001), both a direct and indirect transmission route in bats sharing the same roosts seem possible. Bat astrovirus sequences have been found in feces and urine (Fischer et al. 2017), which are excreted during roost site use. These infectious excretions accumulate at the bottom of the roost sites and remain there after the colony moves to a new communal roost site. Thus, if astroviruses in bats are transmitted indirectly - e.g., via the fecal-oral route as typical for mammalian astroviruses - bats may get infected when using contaminated roost sites even if the virus shedding animals are no longer present. Alternatively, as direct transmission of bat astroviruses cannot be excluded upfront given the close proximity among colony members (Kerth 2008), individuals can be assumed to be at infection risk when closely associating with infected colony members. Despite existing gaps in knowledge regarding their stability and persistence in bat hosts and in the environment, the high detection rate and diversity of bat astroviruses (Chu et al. 2008; Drexler et al. 2011; Fischer et al. 2016, 2017) make them well suited for analyzing virus transmission networks in bat colonies.

Our aim was to examine astrovirus transmission pathways in the social networks of bat colonies. We compared individual association behavior and roost site use (i.e., occupied bat boxes) of a free-ranging maternity colony of Natterer's bats (Myotis nattereri) to data of the bats' detected individual astrovirus status (positive/negative).

During the breeding season in summer, female Natterer's bats form fission-fusion colonies, with subgroups switching day roost sites almost daily (Zeus et al. 2017). Thus, if astroviruses circulate among colony members, their highly dynamic roosting behavior likely results in the contamination of many of the colony's roost sites. Despite the fission-fusion dynamics, the colony network of Natterer's bats is almost fully connected with low modularity and many links per individual (Zeus et al. 2018). Such a social organization, characterized by many social contacts, is presumed to facilitate the spread of pathogens throughout the colony (Keeling 2005; Griffin and Nunn 2012).

We created an undirected, weighted association network based on daily roosting observations in bat boxes (Zeus et al. 2018). To weight ties between individuals, we used the "Simple Ratio Index" (SRI) (Cairns and Schwager 1987; Ginsberg and 
Young 1992) with values ranging between zero (never found roosting together) and one (always found roosting together) (Zeus et al. 2018). Additionally, individual fecal and urine samples were collected and subsequently tested for the presence of astrovirus-related RNA (Fischer et al. 2016).

We examined whether an individual's weighted degree (sum of the edge weights) and its roost site repertoire (number of different roost sites used) influenced its astrovirus status detected by polymerase chain reaction (PCR)-based methods. We expected that individuals closely connected to their colony members (high weighted degree) — and thus more exposed to pathogens carried by other bats - to have a higher infection risk (through direct transmission) than individuals with weaker social bonds (Webber et al. 2016). In cases of indirect transmission, bats that use a higher number of roosts might be more at risk for infections as they may visit more potentially infectious roosts. However, Reckardt and Kerth (2007) showed that the ecologically similar Bechstein's bat significantly preferred roost sites that have not recently been used by colony members over recently occupied ones, possibly due to the avoidance of parasites that remain in the roost sites. Assuming a similar roost selection strategy with respect to virus avoidance in Natterer's bats and taking their frequent roost switching (Zeus et al. 2018) into account, individual bats that switch between a high number of roost sites might instead face a decreased infection risk. They can choose from a greater number of roost sites that have not recently been used and are thus less likely to be contaminated.

Finally, we examined possible astrovirus transmission routes between colony members. If virus transmission occurred predominantly indirectly via contaminated roost sites, we expected more shared sequence types between individuals that used the same roost sites in short temporal succession. Conversely, if astroviruses were mainly transmitted via direct contact, closely associated individuals should share the same virus sequence types more often.

\section{Methods}

\section{Data collection and preparation}

\section{Study site and subjects}

We studied a colony of radio-frequency identification (RFID) tagged female Natterer's bats in a 15-hectare forest patch near the city of Würzburg, Germany, between 19 May and 19 September 2014 on a total of 114 consecutive days.

This maternity colony has been present in the area for many years and the subject of regular study since 2011. The colony forms each year after their members return from their hibernation sites to the area in April and May and disintegrates in late August and September. Every year before females give birth at the end of May as well as between late July and September after the fledging of the offspring, several capture events take place to assess the status of the colony. The timeframe of these events is restricted to the time outside the late gestation period and the main lactation period due to animal welfare regulations. All bats are captured by hand from their day roosts (bat boxes). Individual parameters (e.g., sex and reproductive status) are assessed, and previously unmarked individuals are tagged with a RFID transponder via subcutaneous injection. Moreover, individual swab samples of excreted feces and urine are collected if spontaneously excreted by the individual during the handling process (for details see the paragraph "Detection of astrovirus sequences and definition of sequence type groups").

The regular capture events across many years have resulted in a reliable census of the colony. In 2014, we tagged 15 previously unmarked adult individuals during the capture events in April and May, while we encountered no unmarked adults in August and September. Thus, we concluded that the colony consisted of 82 adult female individuals at the start of the assessed 2014 maternity season. Between May and July, in the midst of maternity season, 10 adults vanished and never reappeared in 2014 or the following years.

Over the course of previous years, 134 bat boxes (type $2 \mathrm{FN}$, diameter $16 \mathrm{~cm}$ and height $36 \mathrm{~cm}$, Schwegler, Germany; hereinafter referred to as "roost sites") have been installed in the study site. The boxes are used regularly as maternity roosts by the Natterer's bats in addition to natural tree cavities and have been monitored with RFID-loggers in previous studies to collect data on roost site use (Zeus et al. 2017, 2018) and association behavior (Zeus et al. 2018).

Although the model of bat boxes used can accommodate at least 70 individuals of Natterer's bats (Zeus et al. 2017), typically a much smaller group of individuals is clustered together at the inner roof of the bat box (Zeus et al. 2017). This type of bat box is equipped with an intermediate floor that has an opening for the bats to pass through when entering or exiting the box. Dropped bat feces and urine will accumulate on that floor or fall through the opening. Thus, even if the bat box has not been occupied for several days, excrements of previous inhabitants will be present, and bats passing through the entrance into the (unoccupied) box will come in contact with these excrements.

Based on the available data, we excluded several individuals or used subsets of the colony for parts of the analysis: All juvenile bats as well as several adult colony members were fully excluded due to insufficient RFID data (see "Data collection and preparation: Social network data"). Individuals with adequate RFID data but no data on viral infection (no sample available or inconclusive PCR results) were included in the calculation of the network parameters and roost site use (see "Data collection and preparation: Social network data" and "Data collection and preparation: Roost site use") for a 
realistic representation of the colony structure. However, due to lack of sample material, they were omitted from the further analysis of astrovirus data.

Only individuals that were successfully sampled and analyzed for virus data and also provided sufficient RFID data were part of the actual analysis (see "Data analysis" section below). None of the bats that vanished during the course of the study were in this category, as they had all disappeared before the sampling for virus data in August and September took place (see "Data collection and preparation: Detection of astrovirus sequences and definition of sequence type groups"). Some of these individuals are, however, included in the calculations for the colony structure, if sufficient RFID data were available prior to their disappearance.

An overview of all colony members that were, at least partially, included in our analyses and their respective available data can be found in Tables S1 and S2 in the supplement.

\section{Social network data}

The undirected social network data are based on the RFID dataset. We excluded nine bats of the colony due to insufficient data, as we found a correlation between the number of observations and the number of different association partners for individuals with less than 10 observations (Zeus et al. 2018). For the remaining 73 adult bats, we analyzed undirected daily roosting associations (simultaneous presence in the roost site) using the R package "asnipe" (Farine 2016). We calculated for each pair of individuals the association indices (hereinafter referred to as "pairwise association strength"), and for each individual the weighted degree (total sum of an individual's pairwise association strength) using the "Simple Ratio Index" (SRI) (Cairns and Schwager 1987; Ginsberg and Young 1992). As previously shown by Zeus et al. (2018), the colony network was almost fully connected (network density, 98.136; mean binary degree, 70.7 with range 63-72) and only consisted of one community (network modularity, 3.552714e-15). Moreover, the pairwise association strength between colony members were rather low (mean SRI-value \pm coefficient of variation: $0.199 \pm 58.048$ ), but associations were non-random (Zeus et al. 2018). On average, an individual shared its roost simultaneously with 15 to 28 other colony members (Zeus et al. 2018).

\section{Roost site use}

Based on the RFID-data, we further assessed each of the individual's roost site repertoire - the number of different bat boxes used by an individual within the study period (Zeus et al. 2018). This was conducted for all 73 bats that were included in the network analysis. The roost site repertoire varied strongly between individuals, ranging from seven to 45 different bat boxes per individual (mean: 27.5; Zeus et al.
2018) of the existing 134 boxes in the study area. The movements of colony members between the bat boxes were high, as an individual bat switched on its roost site on average every 1.4 days (Zeus et al. 2018). The number of different bats per occupied bat box varied between one and 54 (mean 14.7; Zeus et al. 2017 supplement data 2014).

In addition, we calculated for each pair of individuals how often they separately occupied the same roost site within four days after one another (hereinafter referred to as "subsequent sharing of roost sites"). For example, for a given pair consisting of an individual $\mathrm{A}$ and an individual $\mathrm{B}$, we counted each time individual $\mathrm{A}$ was using a roost site within 4 days after individual $\mathrm{B}$ had left that roost site to move to a new day roost. If during this time frame the bat $\mathrm{B}$ returned to the roost site where A was still present-resulting in a direct contact between the two bats - the census was canceled and started again after one of the two bats had moved to another roost site. This was done to exclude periods with direct contact between the respective bats from this dataset of subsequent roost site sharing. We proceeded accordingly for individual B using roost sites after individual $\mathrm{A}$ and subsequently summed the results of both individual A following individual B in its roost site use and vice versa. This yielded an undirected roosting index for each pair of individuals that represents how often they shared the same roost site within four days albeit not simultaneously. The R script for these calculations is available upon request.

The timeframe of four days was chosen following Abad et al. (2001) where the stability of human astroviruses on different surfaces and at different temperature regimes was examined. While displaying strong temperature sensitivity, human astroviruses in a fecal suspension applied on a porous surface (cellulose filter paper) and kept at a temperature of 20 ${ }^{\circ} \mathrm{C}$ remained infective for 7 days (Abad et al. 2001). Although the interior surfaces of the bat boxes were also porous, we assumed that temperature conditions were less stable than in the experimental setup by Abad et al. (2001) and likely exceeded $20{ }^{\circ} \mathrm{C}$ in the summer months. We therefore took a conservative approach and set the timeframe to 4 days. We repeated this analysis for time spans of 1,2 , and 3 days, to test for the sensitivity of our analyses with respect to the time span between events of subsequent sharing of roost sites.

\section{Detection of astrovirus sequences and definition of sequence type groups}

During the capture events, individual fecal and urine samplesif spontaneously excreted by the adult individuals - were collected via swabs and stabilized in 500- $\mu$ l RNAlater ${ }^{\mathrm{TM}}$ buffer (Thermo Fisher Scientific). Due to the small volumes of urine and feces, the sample quantity was not standardized. We therefore cannot exclude the possibility that the amount of viral RNA in a given sample might in some cases be below the 
detection limit. In the lab, the samples were stored at $-20^{\circ} \mathrm{C}$ until analysis. To ensure a temporal context between roost use and associations during the summer and astrovirus infections, we only analyzed samples collected between August and September during seven capture events.

RNA was extracted using the QIAamp Viral RNA Mini Kit (QIAGEN) following the standard protocol. A hemi-nested reverse transcription PCR following the protocol of Chu et al. (2008) targeting the RdRp gene was used. This method has been used as a basis for the detection of astroviruses in different mammalian and avian species in over 100 published studies. Samples showing a single band in the expected size of around 400 nucleotides were purified using the GeneJET PCR Purification Kit (Thermo Fisher Scientific). Sequence analysis of purified RNA fragments was performed via tube read service by Eurofins genomics. A forward read and a reverse read were used to build a consensus sequence, and unambiguous sequence areas were used to identify sequence variants by aligning and comparing them with known bat astrovirus sequences from NCBI GenBank. All PCR analyses were performed in three individual experiments to minimize the rate of ambiguous sequences. Afterwards a consensus sequence of all alignments was built for each sample. Due to the relatively low stability of viral RNA in field samples - and especially in fecal samples as a result of RNA degrading and PCR inhibiting effects in the intestinal surroundings - the triplicate PCR assays did not reveal positive results in all runs. Only samples that tested positive at least two out of three times were counted as positive (detected astrovirus status). Samples that were tested negative in all three runs were counted as negative, while samples with only one positive result were classified as inconclusive and removed from subsequent data analysis. Individuals for which several samples had been collected across the seven different capture events were regarded as astrovirus positive, if at least one of their samples tested positive.

Next, we defined sequence type groups (TG) for the identification of variants in the amplified sequences. This was necessary since we did not have access to the full-length genome data of the astrovirus variants, as the applied PCR protocol leads to the amplification of a 400-bp fragment within the ORF1 (RdRP gene). Our analysis was restricted to this short and comparably highly conserved fragment due to the relatively poor RNA quality in the fecal and urine samples, taking into consideration that we may be missing additional variations outside the analyzed area. The astrovirus genome encodes for three open reading frames ORF1a, ORF1b, and ORF2 which partly overlap in most variants, making the identification of the individual ORFs difficult. We therefore focused our phylogenetic analysis on a highly conserved target region of 60 amino acids (aa) and defined all variants with an identical sequence in this area as one type group (TG). To identify this region, we used the specific 32 aa sequence "WYxxxxxxxxxxxPxGExxxxxxGNPSGQxST," which was present in all of our detected astrovirus fragments for a reliable orientation, and added five aa positions upstream and 23 aa positions downstream of this sequence, adding up to 60 aa (Fig. S1 in supplemental section).

Samples collected and PCR results, individual network parameters of all 73 colony members, and their astrovirus status as well as detected TG are found in Tables S1 and S2. The colony's network structure and astrovirus status are shown in Fig. S2.

All data on association and roost use were recorded automatically; thus, there was little need for blinded methods. During the capture events, sequential sample numbers were assigned to the individual fecal and urine samples for anonymization. Moreover, lab work and field work were conducted by different research teams in different institutes respectively.

\section{Data analysis}

Data analysis was performed in $\mathrm{R}$ version 3.6.2 ( $\mathrm{R}$ Core Team 2016). Only the 42 bats with sufficient association data and that had been successfully sampled and analyzed for virus data were included (compare Table S2: "astrovirus status" listed as "positive" and "negative," bats with "inconclusive" astrovirus status were omitted from the dataset).

The first part of the analysis focused on the association and roosting behavior of the individual bats, and their respective astrovirus status. To assess if an individual's weighted degree and/or its roost site repertoire influenced whether that individual was astrovirus positive, we used a generalized linear model (GLM). For each of the 42 individuals, the presence/ absence of astrovirus RNA was set as response variable, while the individual's weighted degree and roost site repertoire were set as predictors. We conducted the same analysis with eigenvector centrality (a measure of influence; Borgatti 2005) and binary degree (the total number of connections) as the predictor variables (Table S3). However, as both of these additional network parameters were highly correlated with the weighted degree (Kendall rank correlation coefficient (Kendall 1938) weighted degree and eigenvector, 0.973; weighted degree and binary degree, 0.651 ), the results can be found in the supplement (Table S3).

The second part of the analysis examined pairwise relations between colony members. We assessed whether pairs of individuals that strongly associated with each other and/or often shared the same roost site after each other (but not simultaneously) would exhibit the same TGs. We had to limit our dataset to the 30 individuals that were tested astrovirus positive, which amounted to a total of 435 pairs of individuals. Our aim was to examine whether a direct transmission pathway, an indirect transmission pathway, or a combination of both best predicted virus transmission between pairs of individuals. Therefore, we used pairwise association strength as a 
proxy for direct transmission, and subsequent sharing of roost sites (within 1, 2, 3, or 4 days) as a proxy for indirect transmission. If a pair of individuals shared at least one TG, we assumed that an astrovirus variant was transmitted between these bats. Although a pair's association strength and their index of subsequent sharing of roost sites were positively correlated - with correlation strength depending on the selected timeframe for roost site sharing (Spearman's rank correlation, $p<0.001$, $\mathrm{rho}_{1 \text { day }}=0.623, \mathrm{rho}_{2 \mathrm{days}}=0.532, \mathrm{rho}_{3 \text { days }}=$ 0.468, rho $_{4 \text { days }}=0.442$ ) - the scatter plots (see Fig. S3 in the supplement) revealed a broad distribution of data points. This second part of the analysis was calculated via multiple regression quadratic assignment procedures (MRQAP) with double Dekker semipartialling (Dekker et al. 2007) and 1000 permutations using the "sna" package in R (Butts 2016)_following VanderWaal et al. (2014) on social and pathogen transmission networks in giraffes. Virus transmission was tested against each predictor variable - pairwise association strength and subsequent sharing of roost sites - separately and both in combination by using logistic regression models. The selection of the best model was determined using the Akaike information criterion (AIC). Thereby, the model with the lowest AIC was regarded the best unless a simpler model scored a delta AIC (AIC differences, $\triangle \mathrm{AIC}$ ) of less than two in which case the simpler model was preferred (Burnham et al. 2011)

For all predictors to have equal range and variance in models, in both the GLM and MRQAP analyses, the respective predictor variables were rescaled (mean $=0$, standard deviation $=1$ ).

\section{Data availability}

All astrovirus data generated or analyzed are included in here or in the supplement. The social network parameters and roost use data of all individual bats that were used for the GLM analysis are in the supplement. The pairwise association data and daily roosting observations that were used for the MRQAP analysis and the computation of the individual parameters are available from the corresponding author.

\section{Results}

\section{Virus detection rate and type groups}

During our capture events, we collected a total of 75 samples (41 urine and 34 fecal samples). Of these-after conducting three PCR runs per sample - 40 were assessed as astrovirus positive (53.7\% of urine and $52.9 \%$ of fecal samples), 23 astrovirus negative, and 12 inconclusive. The samples originated from 45 members of the colony (62\% of the colony excluding the bats who disappeared). Of these-after analyzing all available samples — we identified 30 bats as astrovirus positive, 12 negative, and three as inconclusive (see Tables S1 and $\mathrm{S} 2$ in the supplement).

Among those 30 positive bats, we found seven different TGs (sequence type groups) with the most common TG being present in $73 \%$ of the positive bats ( 22 individuals), while four TGs were only recovered from one bat each (Fig. 1). In one bat - although astrovirus positive - we were not able to determine a TG as the defined 60 amino acid frame was not covered by sequencing (Table S1 in the supplement). We also had this issue for two other samples from two different individuals. Because these bats were sampled several times, we were able to assign a TG to each of these bats from one of the other samples (Table S1 in the supplement). Overall, we were able to determine TGs for 37 of the 40 astrovirus positive samples.

In two of the astrovirus positive individuals, we detected two TGs simultaneously on a given sampling day. We found one individual to carry two different TGs at different sampling events during the course of the study, and seven bats sampled several times across the study period to switch from negative to positive test results, indicating ongoing virus transmission (see Tables S1 and S2 in the supplement).

\section{Influence on individual astrovirus status}

The GLM assessing relationships between virus detection and the attributes weighted degree (ranging between 3.95 and 20.32; mean weighted degree. 14.32) and roost site repertoire (ranging between seven and 45 different roost sites; mean number of roost sites per individual, 27.5) revealed no significant influence of any parameters (Table 1). Neither an individual's weighted degree nor the number of different roost sites it used during the season had a significant effect on the presence of astroviruses.

\section{Virus transmission}

We found a significant influence of both predictors - association strength and subsequent sharing of roost sites within 4 days - on virus transmission (Table 2, Fig. 2). The model including both variables was best ( $\triangle \mathrm{AIC}=0$, Table 2$)$ to predict virus transmission. It correctly predicted $81.2 \%$ of the total observations from our dataset (accuracy, 0.812; error rate, 0.189; $n=435$ ). Of the pairs that shared the same TG, $81.3 \%$ were correctly predicted by this model (precision, $0.813 ; n=400$ )

The model with just association strength, while inferior to the combined model, had a lower AIC and higher pseudo- $R^{2}$ than the one that solely included subsequent sharing of roost sites within 4 days (Table 2). This indicates that association strength is the more important variable. The models for the other time spans of 1,2 , and 3 days between events of subsequent sharing of roost sites revealed similar results with slightly higher AICs (see Table S4 in the supplement). In the combined model with both independent parameters, the 
Fig. 1 Detection rate of the different virus type groups (TGs) in Natterer's bats from the same colony

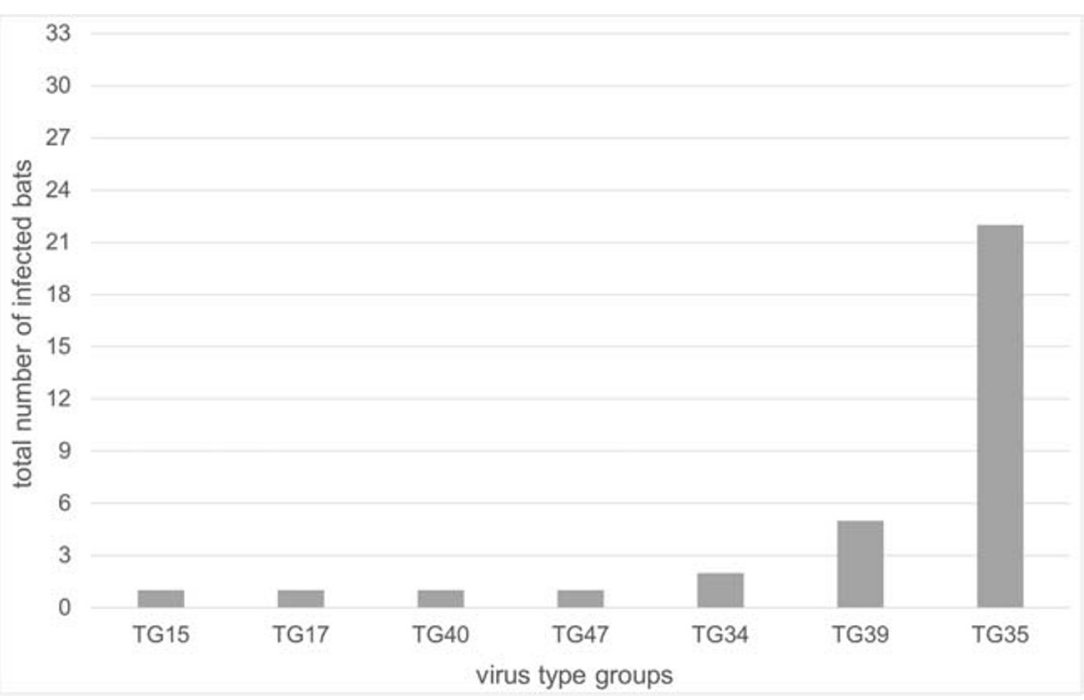

subsequent sharing of roosts was not significant for 1 to 3 days which likely can be attributed to the stronger correlation between the two independent variables (direct association and subsequent roost sharing) in shorter time frames (see also Fig. $\mathrm{S} 3$ in the supplement).

\section{Discussion}

Astrovirus infections are widespread and diverse in mammals (Moser and Schultz-Cherry 2005; De Benedictis et al. 2011), including Natterer's bats (Kemenesi et al. 2014, 2016; Fischer et al. 2016, 2017; Halczok et al. 2017). We found an astrovirus detection rate of $67 \%$ among all sampled individuals. Both sample types (urine and feces) were identified as positives at similar levels, but not always at the same time for the same individual (see Table S1 in the supplement). Moreover, we found variability in the virus detection status of the bats sampled during several capture events. In particular, several individuals changed from negative to positive status during the sampling period in August and September, indicating ongoing transmission in the colony.

Table 1 Results of the GLM to test for a relationship between an individual's detected astrovirus status and its roosting and network parameters

\begin{tabular}{llllll}
\hline Model & Predictor & Estimate & SE & $z$ & $p$ \\
\hline A & Weighted degree & 0.287 & 0.418 & 0.687 & 0.492 \\
B & Roost site repertoire & 0.471 & 0.445 & 1.058 & 0.290 \\
C & Weighted degree & -0.421 & 0.849 & -0.496 & 0.620 \\
& Roost site repertoire & 0.862 & 0.915 & 0.941 & 0.347 \\
\hline
\end{tabular}

The estimate is given with standard error (SE), $z$ value, and the $p$ value
Since there are limited data available from experimental astrovirus infections, and to the best of our knowledge, no experimental astrovirus infection has ever been performed in bats, there is no validation of the diagnostic sensitivity and specificity of our PCR protocol. However, the confirmation of positive PCR results by sequence analysis support the reliability of the positive PCR results. Negative results caused by PCR inhibiting effects in the intestinal surroundings or the degradation of viral RNA in the samples cannot be completely ruled out. Furthermore, we observed seven different astrovirus sequence groups (TGs) among 30 positive bats. Although the analysis of TGs based on a 60 aa fragment cannot completely replace a full genome analysis, we consider this approach a good proxy for our application.

In humans and domestic animals, astrovirus infections are especially common in settings with a high density of hosts, e.g., in schools and nursery homes or in breeding kennels and farms, respectively (Glass et al. 1996; Shan et al. 2011; Grellet et al. 2012). In the highly interconnected colony of Natterer's bats we studied, neither the overall strength of an individual's associations with all other colony members (weighted degree) nor its repertoire of used roost sites had a significant influence on the detected presence of astroviruses in the respective individual. This is surprising given that Webber et al. (2016) using simulated transmission data suggested a relationship between the overall connectivity of colony members and predicted pathogen prevalence. But given the overall high fission-fusion dynamic in our colony-which implies both a high roost switching rate and high potential for environmental transmission - the observed individual variations in association and roosting behavior might have no apparent effect on the astrovirus presence. However, it must be noted that we also ran a GLM to test for the relationship between the successful sampling of an individual (fecal and urine samples) and its roosting and network parameters (weighted degree, roost repertoire). Individuals with low values in the assessed 
Table 2 Reliability of fit statistics and results of the MRQAP models

\begin{tabular}{|c|c|c|c|c|c|c|c|}
\hline \multirow[t]{2}{*}{ Model } & \multirow[t]{2}{*}{ Predictor } & \multirow[t]{2}{*}{ AIC } & \multirow[t]{2}{*}{$\Delta \mathrm{AIC}$} & \multirow{2}{*}{$\begin{array}{l}\text { pseudo- } \\
\mathrm{R}^{2}\end{array}$} & \multicolumn{2}{|l|}{ Estimate } & \multirow[t]{2}{*}{$p$} \\
\hline & & & & & Log odds & Odds ratios & \\
\hline A & "Association" & 431.388 & 10.396 & 0.288 & 0.868 & 2.383 & 0.001 \\
\hline B & "Roost sites" & 440.166 & 19.174 & 0.277 & 0.794 & 2.212 & 0.005 \\
\hline \multirow[t]{2}{*}{$\mathrm{C}$} & "Association" & 420.992 & 0.000 & 0.302 & 0.646 & 1.909 & 0.014 \\
\hline & "Roost sites" & & & & 0.493 & 1.638 & 0.043 \\
\hline
\end{tabular}

Virus transmission (i.e., whether or not a pair of individuals shared the same TG) was set as the response variable. Pairwise association strength ("association") and/or subsequent sharing of roost sites ("roost sites") were set as predictors. Sharing of roost sites in these models was set to a time span within 4 days. To assess the fit of the models, the AIC, $\triangle \mathrm{AIC}$, and pseudo- $R^{2}$ as "(null deviance-residual deviance)/(null deviance-residual deviance + numerator degrees of freedom)" are given. The best model is marked in italics. Model results are shown as the $\log$ odds estimate, the odds ratio estimate, and the $p$ value parameters might have been less frequently present during capture events and thus less likely successfully sampled for virus data. Indeed, we found a significantly positive influence of the individual attributes weighted degree and roost site repertoire on successful sampling (see Table S5 in the supplement). Thus, the observed lack of correlation between detected astrovirus presence and the individual roosting and network parameters could have also been due to a sampling bias. This poses a methodological constraint as, due to animal welfare concerns and regulations, we were not able to make capture attempts until all individuals were caught and successfully sampled, neither was a more invasive sampling method (e.g., via bladder palpation) an option.

Astroviruses are typically transmitted between hosts via the fecal-oral route (Mendenhall et al. 2015). Regarding the results of our MRQAP analysis, we suggest that in Natterer's bats indirect transmission via contaminated roost sites might play a significant role in astrovirus infections (model B, Table 2), with the virus likely to persist for several days in the environment (Abad et al. 2001). Bats display two main behavioral mechanisms of parasite

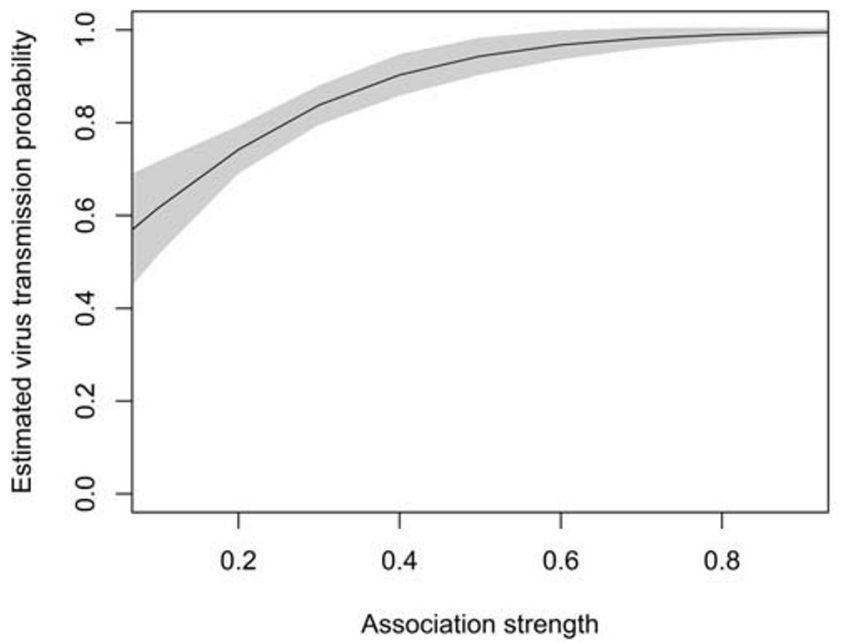

Fig. 2 Relationship between the estimated probability of sharing at least one TG (virus type group) and the predictors pairwise association strength (right) as well as subsequent sharing of roost sites within 4 days (left), respectively, based on our best fitted model (model C, Table 2). The value and pathogen avoidance: roost site selection and roost switching (Lewis 1995; Reckardt and Kerth 2007). Bechstein's bats prefer roost sites that have not been used previously — or at least not in the current year- over recently occupied ones, which are potentially infected with parasites and/or other pathogens (Reckardt and Kerth 2007). Moreover, in both Bechstein's bats (Kerth et al. 2000) and brown long-eared bats (Zeus et al. 2017), different conspecific colonies do not share roost sites, which likely reduces the exposure risk of species-specific ectoparasites. Many forest-living bat species (Kerth and König 1999; O’Donnell 2000; Garroway and Broders 2007; Popa-Lisseanu et al. 2008), including Natterer's bats (Zeus et al. 2017, 2018), switch roost sites almost daily and frequently split into subgroups. This "fission-fusion" behavior has been assumed to be a mechanism to avoid parasites and other pathogens that may be present in the roost sites (Reckardt and Kerth 2007; Kashima et al. 2013; Webber et al. 2016). On the other hand, the frequent switching between many roost sites might also facilitate pathogen transmission, as it would result in the contamination of a high number of different roost sites. Besides an indication for indirect

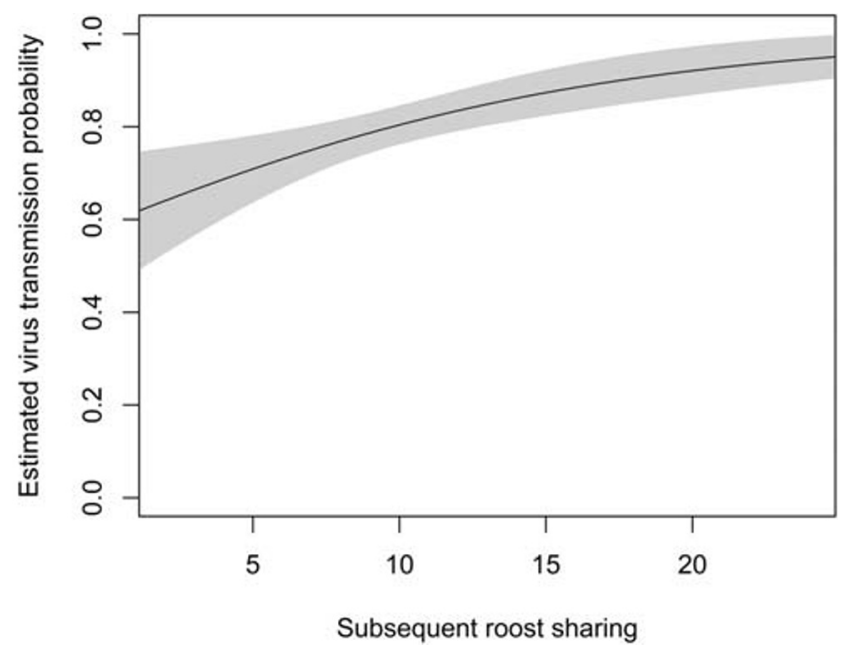

of the respective other predictor is set as the mean ("subsequent sharing of roost sites", mean number of roost sites $=9.8$; "pairwise association strength", mean association strength $=0.26$ ). Shading indicates a $95 \%$ confidence interval around the regression line 
transmission, the MRQAP models provide evidence for a direct astrovirus transmission in Natterer's bats via association (model A, Table 2). This is not surprising, given the close contact between roost mates in bat maternity colonies in general (Kerth 2008). In addition, if two bats are simultaneously roosting in the same bat boxes, they are also exposed to the same (potentially contaminated) excrement when passing through the entrance of the respective bat boxes. As our combined model with both parameters (model C, Table 2) was the best fit, we conclude that both direct and indirect transmission of astroviruses likely occurs in bat roosts.

While our best model correctly predicted $81.2 \%$ of our observations, none of our three models fully explained the data. This might be due to constraints on our field data- e.g., only $62 \%$ of individuals were successfully sampled for virus data, the lack of temporal resolution of the virus data, and the clear predominance of one TG in the colony. This predominance was unexpected, given the high diversity of astroviruses in bats (Chu et al. 2008; Drexler et al. 2011; Fischer et al. 2016, 2017). All these may limit the predictive power of our model, which we nevertheless consider as a good predictor given the natural limitations of a field study.

In addition, it was impossible to assess the influence of both transmission pathways independently. A spatial separation of direct and indirect transmission was not given, as both are expected to take place in temporal succession in any occupied bat box. Furthermore, due to the association and roosting behavior of the colony, most of the pairs of individuals engaged in both direct and indirect contact with each other. Only $0.5 \%$ of the pairs of individuals (two of 435) in our dataset for the MRQAP analysis were associated but never shared a roost within 1 to 4 days after one another; $0.2 \%$ (one of 435 ) were never associated but subsequently shared a roost in the given timeframe. Such caveats are common when working with elusive, protected, and free-ranging animals. However, we are convinced that datasets like ours are invaluable for future research both for the design of follow-up field studies and for setting refined parameters in simulation studies exploring virus transmission in bat colonies.

To conclude, the precise factors influencing the presence of astroviruses in Natterer's bats need further evaluation, as we found that neither an individual's weighted degree nor the number of different roost sites it used during the season played a significant role. At the same time, we found implications for both direct and indirect transmission routes, although more - yet unknown - factors also seem to be involved. Overall, our study provides novel insights into the transmission of astroviruses within the social network of free-ranging bats.

Supplementary Information The online version contains supplementary material available at https://doi.org/10.1007/s00265-020-02932-y.

Acknowledgments Pia Baumgärtner supported the PCR-analyses of the samples. We thank the local forestry and conservation departments for support, Markus Melber for help in the field, and Dr. Jaap van Schaik, as well as two anonymous reviewers for helpful comments on the manuscript.

Funding Open Access funding enabled and organized by Projekt DEAL. This work was supported by the Deutsche Forschungsgemeinschaft DFG (KE 746/6-1) within the priority program "Ecology and species barriers in emerging viral diseases (SPP 1596)" and the DFG Research training Group "Biological Responses to Novel and Changing Environments" (RTG 2010).

\section{Compliance with ethical standards}

Conflict of interest The authors declare that they have no competing interests.

Ethical approval All applicable international, national, and/or institutional guidelines for the care and use of animals were followed. Handling and tagging of the bats were conducted under the permits for species protection (55.1-8642.01-2/00) and animal welfare (55.22531.01-47/11 and 55.2-2532-2-20) that had been issued by the government of Lower Franconia.

Open Access This article is licensed under a Creative Commons Attribution 4.0 International License, which permits use, sharing, adaptation, distribution and reproduction in any medium or format, as long as you give appropriate credit to the original author(s) and the source, provide a link to the Creative Commons licence, and indicate if changes were made. The images or other third party material in this article are included in the article's Creative Commons licence, unless indicated otherwise in a credit line to the material. If material is not included in the article's Creative Commons licence and your intended use is not permitted by statutory regulation or exceeds the permitted use, you will need to obtain permission directly from the copyright holder. To view a copy of this licence, visit http://creativecommons.org/licenses/by/4.0/.

\section{References}

Abad FX, Villena C, Guix S, Caballero S, Pintó RM, Bosch A (2001) Potential role of fomites in the vehicular transmission of human astroviruses. Appl Environ Microbiol 67:3904-3907. https://doi. org/10.1128/AEM.67.9.3904-3907.2001

Amici F, Aureli F, Call J (2008) Fission-fusion dynamics, behavioral flexibility, and inhibitory control in primates. Curr Biol 18:14151419. https://doi.org/10.1016/j.cub.2008.08.020

Amoroso MG, Russo D, Lanave G, Cistrone L, Pratelli A, Martella V, Galiero G, Decaro N, Fusco G (2018) Detection and phylogenetic characterization of astroviruses in insectivorous bats from CentralSouthern Italy. Zoonoses Public Health 65:702-710. https://doi.org/ 10.1111/zph. 12484

Archie EA, Moss CJ, Alberts SC (2006) The ties that bind: genetic relatedness predicts the fission and fusion of social groups in wild African elephants. Proc R Soc Lond B 273:513-522. https://doi. org/10.1098/rspb.2005.3361

Baker ML, Schountz T, Wang L-F (2013) Antiviral immune responses of bats: a review. Zoonoses Public Health 60:104-116. https://doi.org/ 10.1111/j.1863-2378.2012.01528.x

Blomström A-L, Widén F, Hammer A-S, Belák S, Berg M (2010) Detection of a novel astrovirus in brain tissue of mink suffering from shaking mink syndrome by use of viral metagenomics. J Clin Microbiol 48:4392-4396. https://doi.org/10.1128/JCM.01040-10 
Borgatti SP (2005) Centrality and network flow. Soc Networks 27:55-71 Bouzalas IG, Wüthrich D, Walland J, Drögemüller C, Zurbriggen A, Vandevelde M, Oevermann A, Bruggmann R, Seuberlich T (2014) Neurotropic astrovirus in cattle with nonsuppurative encephalitis in Europe. J Clin Microbiol 52:3318-3324. https://doi.org/10. 1128/JCM.01195-14

Burnham KP, Anderson DR, Huyvaert KP (2011) AIC model selection and multimodel inference in behavioral ecology: some background, observations, and comparisons. Behav Ecol Sociobiol 65:23-35. https://doi.org/10.1007/s00265-010-1029-6

Butts DR (2016) Sna: tools for social network analysis. R package version 1.0. https://CRAN.R-project.org/package=asnipe. Accessed 02 Feb 2020

Cairns SJ, Schwager SJ (1987) A comparison of association indices. Anim Behav 35:1454-1469. https://doi.org/10.1016/S00033472(87)80018-0

Calisher CH, Childs JE, Field HE, Holmes KV, Schountz T (2006) Bats: important reservoir hosts of emerging viruses. Clin Microbiol Rev 19:531-545. https://doi.org/10.1128/CMR.00017-06

Carter KD, Seddon JM, Frère CH, Carter JK, Goldizen AW (2013) Fission-fusion dynamics in wild giraffes may be driven by kinship, spatial overlap and individual social preferences. Anim Behav 85: 385-394. https://doi.org/10.1016/j.anbehav.2012.11.011

Chan JF-W, To KK-W, Tse H, Jin D-Y, Yuen K-Y (2013) Interspecies transmission and emergence of novel viruses: lessons from bats and birds. Trends Microbiol 21:544-555. https://doi.org/10.1016/j.tim. 2013.05.005

Chu DKW, Poon LLM, Guan Y, Peiris JSM (2008) Novel astroviruses in insectivorous bats. J Virol 82:9107-9114. https://doi.org/10.1128/ JVI.00857-08

Cortez V, Meliopoulos VA, Karlsson EA, Hargest V, Johnson C, Schultz-Cherry S (2017) Astrovirus biology and pathogenesis. Annu Rev Virol 4:327-348. https://doi.org/10.1146/annurevvirology-101416-041742

De Benedictis P, Schultz-Cherry S, Burnham A, Cattoli G (2011) Astrovirus infections in humans and animals - molecular biology, genetic diversity, and interspecies transmissions. Infect Genet Evol 11:1529-1544. https://doi.org/10.1016/j.meegid.2011.07.024

Dekker D, Krackhardt D, Snijders TAB (2007) Sensitivity of MRQAP tests to collinearity and autocorrelation conditions. Psychometrika 72:563-581. https://doi.org/10.1007/s11336-007-9016-1

Drexler JF, Corman VM, Wegner T, Tateno AF, Zerbinati RM, GlozaRausch F, Seebens A, Müller MA, Drosten C (2011) Amplification of emerging viruses in a bat colony. Emerg Infect Dis 17:449-456. https://doi.org/10.3201/eid1703.100526

Farine DR (2016) Asnipe: animal social network inference and permutations for ecologists. Version R package version 1.0. https://CRAN. R-project.org/package=asnipe. Accessed 02 Feb 2020

Fischer K, Zeus V, Kwasnitschka L, Kerth G, Haase M, Groschup MH, Balkema-Buschmann A (2016) Insectivorous bats carry host specific astroviruses and coronaviruses across different regions in Germany. Infect Genet Evol 37:108-116. https://doi.org/10.1016/j. meegid.2015.11.010

Fischer K, Pinho dos Reis V, Balkema-Buschmann A (2017) Bat astroviruses: towards understanding the transmission dynamics of a neglected virus family. Viruses 9:34. https://doi.org/10.3390/ v9020034

Garroway CJ, Broders HG (2007) Nonrandom association patterns at northern long-eared bat maternity roosts. Can J Zool 85:956-964. https://doi.org/10.1139/Z07-079

Ginsberg JR, Young TP (1992) Measuring association between individuals or groups in behavioural studies. Anim Behav 44:377-379. https://doi.org/10.1016/0003-3472(92)90042-8

Glass RI, Noel J, Mitchell D, Herrmann JE, Blacklow NR, Pickering LK, Dennehy P, Ruiz-Palacios G, De Guerrero ML, Monroe SS (1996)
The changing epidemiology of astrovirus-associated gastroenteritis: a review. Arch Virol Suppl 12:287-300

Grellet A, De Battisti C, Feugier A, Pantile M, Marciano S, Grandjean D, Cattoli G (2012) Prevalence and risk factors of astrovirus infection in puppies from French breeding kennels. Vet Microbiol 157:214 219. https://doi.org/10.1016/j.vetmic.2011.11.012

Griffin RH, Nunn CL (2012) Community structure and the spread of infectious disease in primate social networks. Evol Ecol 26:779-800

Halczok TK, Fischer K, Gierke R, Zeus V, Meier F, Treß C, BalkemaBuschmann A, Puechmaille SJ, Kerth G (2017) Evidence for genetic variation in Natterer's bats (Myotis nattereri) across three regions in Germany but no evidence for co-variation with their associated astroviruses. BMC Evol Biol 17:5. https://doi.org/10.1186/s12862016-0856-0

Imada T, Yamaguchi S, Mase M, Tsukamoto K, Kubo M, Morooka A (2000) Avian nephritis virus (ANV) as a new member of the family Astroviridae and construction of infectious ANV cDNA. J Virol 74: 8487-8493. https://doi.org/10.1128/JVI.74.18.8487-8493.2000

Kashima K, Ohtsuki H, Satake A (2013) Fission-fusion bat behavior as a strategy for balancing the conflicting needs of maximizing information accuracy and minimizing infection risk. J Theor Biol 318:101109. https://doi.org/10.1016/j.jtbi.2012.10.034

Keeling M (2005) The implications of network structure for epidemic dynamics. Theor Popul Biol 67:1-8

Kemenesi G, Dallos B, Görföl T, Boldogh S, Estók P, Kurucz K, Kutas A, Földes F, Oldal M, Németh V, Martella V, Bányai K, Jakab F (2014) Molecular survey of RNA viruses in Hungarian bats: discovering novel astroviruses, coronaviruses, and caliciviruses. VectorBorne Zoonotic Dis 14:846-855. https://doi.org/10.1089/vbz.2014. 1637

Kemenesi G, Gellért Á, Dallos B, Görföl T, Boldogh S, Estók P, Marton S, Oldal M, Martella V, Bányai K, Jakab F (2016) Sequencing and molecular modeling identifies candidate members of Caliciviridae family in bats. Infect Genet Evol 41:227-232. https://doi.org/10. 1016/j.meegid.2016.04.004

Kendall MG (1938) A new measure of rank correlation. Biometrika 30 (1-2):81-93. https://doi.org/10.1093/biomet/30.1-2.81

Kerth G (2008) Causes and consequences of sociality in bats. BioScience 58:737-746. https://doi.org/10.1641/B580810

Kerth G, König B (1999) Fission, fusion and nonrandom associations in female Bechstein's bats (Myotis bechsteinii). Behaviour 136:11871202. https://doi.org/10.1163/156853999501711

Kerth G, Mayer F, König B (2000) Mitochondrial DNA (mtDNA) reveals that female Bechstein's bats live in closed societies. Mol Ecol 9: 793-800. https://doi.org/10.1046/j.1365-294x.2000.00934.x

Klimpel S, Mehlhorn H (eds) (2014) Bats (Chiroptera) as vectors of diseases and parasites. Springer, Berlin

Krause J, Ruxton GD (eds) (2002) Living in groups. Oxford University Press, Oxford

Kunz TH, Braun de Torrez E, Bauer D, Lobova T, Fleming TH (2011) Ecosystem services provided by bats. Ann N Y Acad Sci 1223:138. https://doi.org/10.1111/j.1749-6632.2011.06004.x

Lewis SE (1995) Roost fidelity of bats: a review. J Mammal 76:481-496. https://doi.org/10.2307/1382357

Li L, Diab S, McGraw S, Barr B, Traslavina R, Higgins R, Talbot T, Blanchard P, Rimoldi G, Fahsbender E, Page B, Phan TG, Wang C, Deng X, Pesavento P, Delwart E (2013) Divergent astrovirus associated with neurologic disease in cattle. Emerg Infect Dis 19:13851392. https://doi.org/10.3201/eid1909.130682

Loehle C (1995) Social barriers to pathogen transmission in wild animal populations. Ecology 76:326-335. https://doi.org/10.2307/1941192

Luis AD, Hayman DTS, O'Shea TJ et al (2013) A comparison of bats and rodents as reservoirs of zoonotic viruses: are bats special? Proc R Soc B 280:20122753. https://doi.org/10.1098/rspb.2012.2753 
Mendenhall IH, Smith GJD, Vijaykrishna D (2015) Ecological drivers of virus evolution: astrovirus as a case study. J Virol 89:6978-6981. https://doi.org/10.1128/JVI.02971-14

Moser LA, Schultz-Cherry S (2005) Pathogenesis of astrovirus infection. Viral Immunol 18:4-10. https://doi.org/10.1089/vim.2005.18.4

O'Donnell CFJ (2000) Cryptic local populations in a temperate rainforest bat Chalinolobus tuberculatus in New Zealand. Anim Conserv 3: 287-297. https://doi.org/10.1111/j.1469-1795.2000.tb00114.x

Omatsu T, Watanabe S, Akashi H, Yoshikawa Y (2007) Biological characters of bats in relation to natural reservoir of emerging viruses. Comp Immunol Microbiol Infect Dis 30:357-374. https://doi.org/ 10.1016/j.cimid.2007.05.006

Padmanabhan A, Hause BM (2016) Detection and characterization of a novel genotype of porcine astrovirus 4 from nasal swabs from pigs with acute respiratory disease. Arch Virol 161:2575-2579. https:// doi.org/10.1007/s00705-016-2937-1

Parra GJ, Corkeron PJ, Arnold P (2011) Grouping and fission-fusion dynamics in Australian snubfin and Indo-Pacific humpback dolphins. Anim Behav 82:1423-1433. https://doi.org/10.1016/j. anbehav.2011.09.027

Pfaff F, Schlottau K, Scholes S, Courtenay A, Hoffmann B, Höper D, Beer M (2017) A novel astrovirus associated with encephalitis and ganglionitis in domestic sheep. Transbound Emerg Dis 64:677-682. https://doi.org/10.1111/tbed.12623

Plowright RK, Eby P, Hudson PJ, Smith IL, Westcott D, Bryden WL, Middleton D, Reid PA, McFarlane RA, Martin G, Tabor GM, Skerratt LF, Anderson DL, Crameri G, Quammen D, Jordan D, Freeman P, Wang LF, Epstein JH, Marsh GA, Kung NY, McCallum H (2015) Ecological dynamics of emerging bat virus spillover. Proc R Soc B 282:20142124. https://doi.org/10.1098/ rspb.2014.2124

Popa-Lisseanu AG, Bontadina F, Mora O, Ibáñez C (2008) Highly structured fission-fusion societies in an aerial-hawking, carnivorous bat. Anim Behav 75:471-482. https://doi.org/10.1016/j.anbehav.2007. 05.011

R Core Team (2016) R: a language and environment for statistical computing. R Foundation for Statistical Computing, Vienna. http:// www.R-project.org. Accessed 02 Feb 2020

Reckardt K, Kerth G (2007) Roost selection and roost switching of female Bechstein's bats (Myotis bechsteinii) as a strategy of parasite avoidance. Oecologia 154:581-588. https://doi.org/10.1007/ s00442-007-0843-7

Schlottau K, Schulze C, Bilk S, Hanke D, Höper D, Beer M, Hoffmann B (2016) Detection of a novel bovine astrovirus in a cow with encephalitis. Transbound Emerg Dis 63:253-259. https://doi.org/10.1111/ tbed.12493

Shan T, Li L, Simmonds P, Wang C, Moeser A, Delwart E (2011) The fecal virome of pigs on a high-density farm. J Virol 11:1169711708
Singh A, Mor SK, Jindal N, Patnayak D, Sobhy NM, Luong NT, Goyal SM (2016) Detection and molecular characterization of astroviruses in turkeys. Arch Virol 161:939-946. https://doi.org/10.1007/ s00705-016-2753-7

Smith JE, Kolowski JM, Graham KE, Dawes SE, Holekamp KE (2008) Social and ecological determinants of fission-fusion dynamics in the spotted hyaena. Anim Behav 76:619-636. https://doi.org/10. 1016/j.anbehav.2008.05.001

Sundaresan SR, Fischhoff IR, Dushoff J, Rubenstein DI (2007) Network metrics reveal differences in social organization between two fission-fusion species, Grevy's zebra and onager. Oecologia 151: 140-149. https://doi.org/10.1007/s00442-006-0553-6

Taylor LH, Latham SM, Mark EJ (2001) Risk factors for human disease emergence. Philos Trans R Soc B 356:983-989

VanderWaal KL, Atwill ER, Isbell LA, McCowan B (2014) Linking social and pathogen transmission networks using microbial genetics in giraffe (Giraffa camelopardalis). J Anim Ecol 83:406-414. https://doi.org/10.1111/1365-2656.12137

Webber QMR, Brigham RM, Park AD, Gillam EH, O'Shea TJ, Willis CKR (2016) Social network characteristics and predicted pathogen transmission in summer colonies of female big brown bats (Eptesicus fuscus). Behav Ecol Sociobiol 70:701-712. https://doi. org/10.1007/s00265-016-2093-3

Wood JLN, Leach M, Waldman L, MacGregor H, Fooks AR, Jones KE, Restif O, Dechmann D, Hayman DTS, Baker KS, Peel AJ, Kamins AO, Fahr J, Ntiamoa-Baidu Y, Suu-Ire R, Breiman RF, Epstein JH, Field HE, Cunningham AA (2012) A framework for the study of zoonotic disease emergence and its drivers: spillover of bat pathogens as a case study. Philos Trans R Soc B 367:2881-2892. https:// doi.org/10.1098/rstb.2012.0228

Zeus VM, Puechmaille SJ, Kerth G (2017) Conspecific and heterospecific social groups affect each other's resource use: a study on roost sharing among bat colonies. Anim Behav 123:329-338. https://doi.org/10.1016/j.anbehav.2016.11.015

Zeus VM, Reusch C, Kerth G (2018) Long-term roosting data reveal a unimodular social network in large fission-fusion society of the colony-living Natterer's bat (Myotis nattereri). Behav Ecol Sociobiol 72:99

Zhao W, Zhu AL, Yu Y, Yuan CL, Zhu CX, Yang ZB, Cui L, Hua XG (2011) Complete sequence and genetic characterization of pigeon avian nephritis virus, a member of the family Astroviridae. Arch Virol 156:1559-1565. https://doi.org/10.1007/s00705-011-1034-8

Publisher's note Springer Nature remains neutral with regard to jurisdictional claims in published maps and institutional affiliations. 